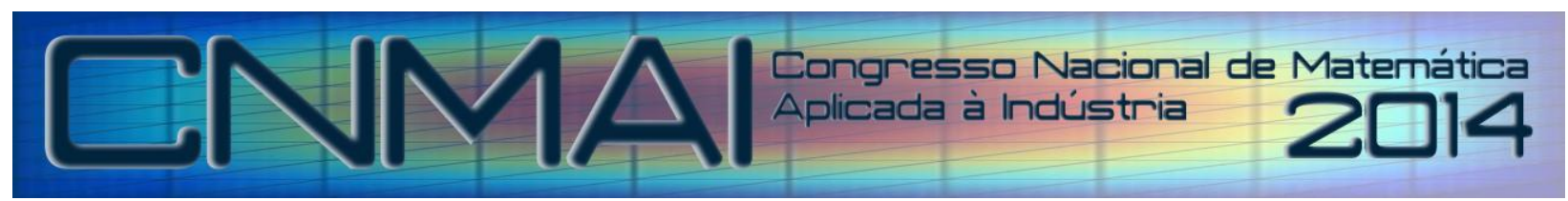

18 a 21 de novembro de 2014, Caldas Novas - Goiás

\title{
MÉTODOS DE LAGRANGEANO AUMENTADO E GRADIENTE ESPECTRAL PROJETADO APLICADOS NA RESOLUÇÃO DO PROBLEMA DO DESPACHO HIDROTÉRMICO
}

\author{
Luiz Carlos Matioli, matioli@ufpr.br ${ }^{1}$ \\ Débora Cíntia Marcilio, deboramarcilio@gmail.com² \\ ${ }^{1}$ Universidade Federal do Paraná - Departamento de Matemática - 81531-990, Centro Politécnico, Curitiba, PR
${ }^{2}$ Universidade Federal do Paraná - PPGMNE - 81531-990, Centro Politécnico, Curitiba, PR
}

Resumo: Nesse trabalho foi desenvolvida uma metodologia de determinação de despacho hidrotérmico, que utiliza programação não linear com modelagem estocástica implícita, adotando representação detalhada das usinas hidroelétricas e termoelétricas. A modelagem do problema do despacho hidrotérmico resulta em um problema de programação não linear de grande porte, não convexo, diferenciável e com restrições não lineares de igualdade, lineares de igualdade e de desigualdade e de caixa. Assim, foi desenvolvida uma metodologia baseada no método de Lagrangeano Aumentado e Gradiente Espectral Projetado, que são conhecidos por possuirem boas propriedades de convergência, robustez, solidez, de fácil implementação e necessitarem apenas de informações de primeira ordem. Para validar a metodologia, foi utilizado um sistema bem representativo do SIN-Sistema Interligado Nacional, com 111 usinas hidráulicas e 32 usinas térmicas, distribuídas em cinco subsistemas. Os resultados obtidos mostraram-se satisfatórios, tanto sob a ótica do problema do despacho quanto do desempenho computacional. Dito isso, a metodologia desenvolvida mostrou-se promissora na determinação de despachos econômicos, pois apresentou resultados coerentes com a realidade e os métodos de Lagrangeano Aumentado e Gradiente Espectral Projetado mostraram se robustos e estáveis.

Palavras-chave: Lagrangeano Aumentado, Gradiente Espectral Projetado, Despacho hidrotérmico, Otimização.

\section{INTRODUÇÃO}

O problema do despacho hidrotérmico consiste na determinação de uma política operativa para determinar a geração de energia de usinas hidroelétricas e termoelétricas, de maneira a atender a demanda com o menor custo esperado de operação.

Atualmente, o Operador Nacional do Sistema Elétrico (ONS) utiliza uma cadeia de softwares desenvolvida pelo Centro de Pesquisas de Energia Elétrica (CEPEL), onde o modelo principal é o NEWAVE que trata da otimização do despacho hidrotérmico no médio prazo. O NEWAVE foi desenvolvido com base na tecnologia de Programação Dinâmica Dual Estocástica (PDDE) (Pereira, 1989 e Pereira et al., 1985) cuja base consiste na decomposição de Benders (Benders, 1962) e na hipótese simplificadora de reservatórios equivalentes. No entanto deve-se ressaltar que desde a criação da primeira versão desses softwares, o Setor Elétrico Brasileiro (SEB) sofreu grandes transformações. O SEB era predominantemente estatal e centralizado, com forte predominância hidrelétrica e com uma folga na geração que permitia acomodar as imprecisões decorrentes das linearizações adotadas.

Dessa forma, considera-se de grande relevância para o SEB a pesquisa envolvendo o desenvolvimento de outros modelos de otimização do despacho hidrotérmico, que melhor se adeque ao cenário atual, visando atender ao mercado de energia elétrica com o menor custo possível e garantindo a oferta futura de recursos energéticos.

O problema do despacho hidrotérmico consiste na otimização de quais usinas devem operar e quais devem ficar de reserva, de modo a preservar o volume dos reservatórios para serem utilizados quando necessário, ou seja, a principal decisão é quanto se deve despachar em cada usina hidrelétrica. Se a decisão tomada for utilizar a água no presente, (despachando as usinas hidrelétricas) e no futuro as afluências forem baixas, ter-se-á um custoso déficit de energia. Por outro lado se a decisão for de economizar água (despachar as usinas térmicas) e as afluências futuras forem altas, o resultado será em vertimento de água, o que significa que não foi aproveitado todo o potencial hidráulico do sistema.

A proposta deste trabalho tem como objetivo otimizar o problema do despacho hidrotérmico formulado como um problema de otimização não linear, tendo como objetivo a minimização dos custos operacionais da geração térmica e do custo de eventuais déficits de energia. As restrições consideradas são o atendimento a demanda, o balanço hídrico e a defluência mínima total para o reservatório. Adicionalmente, são consideradas as restrições operativas que estão representadas pelos limites inferior e superior das variáveis.

A modelagem do despacho hidrotérmico recai em um problema de otimização não linear, de grande porte, não convexo e diferenciável, cuja solução depende de métodos de otimização robustos. Este problema tem sido alvo 
constante de pesquisa e versões iniciais do desenvolvimento proposto foram mostradas nos artigos (Kleina et al, $2012 \mathrm{e}$ Marcilio et al , 2012), onde foi apresentada a modelagem do problema do despacho hidrotérmico, o uso do Método de Pontos Interiores aplicado ao problema do despacho hidrotérmico (Kleina et al , 2012) e uma comparação entre os métodos Lagrangeano Aumentado e Pontos Interiores (Marcilio et al , 2012). O objetivo do presente trabalho é apresentar a utilização do método de Lagrangeano Aumentado juntamente com o SPG - Gradiente Espectral Projetado, que são métodos conhecidos na literatura por resolver problemas com as características do problema do despacho hidrotérmico. A metodologia adotada para a utilização do método de Lagrangeano Aumentado se baseia nos artigos (Birgin e Martinez, 2000, Birgin e Martinez, 2001 e Birgin e Martinez, 2011).

Além disso, foram levadas em consideração as incertezas relativas às afluências futuras para cada reservatório, através da modelagem estocástica implícita. Portanto, o problema do despacho hidrotérmico é um problema estocástico, que ao longo da história vem sendo resolvido por técnicas de otimização estocástica que enfrentam alguns desafios como, por exemplo, o problema da dimensionalidade. Nesse trabalho, optou-se por resolver o problema pela modelagem estocástica implícita, onde os problemas do despacho hidrotérmico são otimizados de forma determinística.

\section{PROBLEMA DO DESPACHO HIDROTÉRMICO}

O modelo considerado na otimização do despacho hidrotérmico consiste na minimização do valor presente dos custos de geração térmica e de déficit. O custo de geração térmica é uma função que representa o custo de cada usina térmica para cada período e é aproximada por um polinômio de grau dois. O custo de déficit é uma função que representa o custo de déficit do subsistema, ou seja, representa o impacto causado pelo não suprimento da demanda de energia e é aproximado por um polinômio de segundo grau.

As restrições levam em consideração as condições operativas das usinas, o balanço hídrico, o atendimento a demanda, a defluência mínima total dos reservatórios e limites das variáveis.

Vale ressaltar que a modelagem do despacho hidrotérmico utilizada nessa pesquisa leva em consideração tanto as usinas hidrelétricas quando as usinas termelétricas de forma individualizada, agregando apenas as usinas pertencentes a um mesmo subsistema na restrição de atendimento a demanda. Uma maior discussão sobre a modelagem do despacho hidrotérmico pode ser encontrada em (Kleina et al , 2012 e Marcilio et al , 2012)

O problema do despacho hidrotérmico, com as características abordadas, quando escrito matematicamente tem o seguinte formato:

$\begin{array}{cc}\text { minimizar } & f(x) \\ \text { sujeito a } & g(x)=0 \\ & A x=b \\ & C x \leq c \\ & l \leq x \leq u\end{array}$

em que $f: \mathbb{R}^{n} \rightarrow \mathbb{R}$ é a função objetivo (não linear), $f: \mathbb{R}^{n} \rightarrow \mathbb{R}^{m}$ representa as restrições não lineares que correspondem ao atendimento a demanda e a geração fixada da usina, $A x=b$ e $C x \leq c$ são restrições lineares que representam, respectivamente, o balanço hídrico e defluência total, $A, C \in \mathbb{R}^{p \times n}, b, c \in \mathbb{R}^{p \times 1}$ e $l \leq x \leq u$ representa os limites das variáveis (também chamadas de restrições de canalização ou caixa), sendo $l, x, u \in \mathbb{R}^{n}$. O vetor $x$ é a variável de decisão, que no caso do problema de despacho hidrotérmico envolve: geração térmica, vazões vertida e turbinada, volume do reservatório, intercâmbio de energia entre subsistemas e déficit.

\section{LAGRANGEANO AUMENTADO}

Métodos de Lagrangeanos Aumentados (Nocedal, 2005 e Luenberger, 2005) são iterativos e baseados em penalização e a cada iteração transformam o problema original restrito em um subproblema irrestrito. Para tanto a estratégia utilizada é a de penalização. As restrições consideradas de difícil trato são acrescidas à função objetivo juntamente com um parâmetro de penalidade e os multiplicadores de Lagrange.

Assim, o método é iterativo e a cada iteração resolve um problema irrestrito, gerado pela penalização das restrições. O critério de convergência utilizado nesse processo é a satisfação das condições de Karush Kuhn Tucker do problema original, e a cada passo em que esses critérios não forem satisfeitos o parâmetro de penalidade é atualizado.

O método de Lagrangeano Aumentado implementado emprega a função penalidade definida por Powell, Hestenes e Rockafellar (1973), as atualizações dos multiplicadores de Lagrange são realizadas de maneira usual forçando satisfazer as condições de KKT. A atualização do parâmetro de penalidade se baseia na metodologia adotada no artigo (Birgin, 2011), onde os autores monitoram o atendimento as restrições, para aumentar, diminuir ou manter constante esse parâmetro.

A metodologia adotada para a utilização do método de Lagrangeano Aumentado se baseia nos artigos (Birgin e Martinez, 2000 e Birgin e Martinez, 2001), em que a cada iteração se penalizam as restrições lineares de desigualdade, 
de igualdade e não lineares de desigualdade. Dessa maneira, na iteração $k$ do método de Lagrangeano Aumentado são conhecidos os multiplicadores de Lagrange $\lambda^{k}$ e $\mu^{k}$ associados, respectivamente, às restrições de igualdade e de desigualdade, o parâmetro de penalidade $\rho^{k}$. O próximo iterando $x^{k+1}$ é determinado resolvendo se o seguinte subproblema:

$$
\begin{array}{cc}
\text { minimizar } & L\left(x, \lambda^{k}, \mu^{k}, \rho^{k}\right) \\
\text { sujeito a } & l \leq x \leq u
\end{array}
$$

Para resolver o subproblema (2) é utilizado o método de Gradiente Espectral Projetado que é descrito na sequência.

\section{GRADIENTE ESPECTRAL PROJETADO}

O método Gradiente Espectral Projetado, também conhecido como SPG (da sigla em Inglês para Spectral Projected Gradient), se origina do principio que o método do Gradiente Projetado é de fácil implementação e bastante eficiente. A partir desse conceito, os autores (Birgin e Martinez, 2000) combinaram esse método a dois ingredientes de otimização. Primeiro, estenderam as estratégias de globalização típicas associadas a estes métodos para o esquema de busca linear não monótona desenvolvido por Grippo, Lampariello e Lucidi (1986) para o método de Newton, o que representou uma grande melhoria em relação aos métodos de gradientes projetados tradicionais. Segundo, propuseram a associação do passo espectral, introduzido por Barzilai e Borwein (1988) e analisado por Raydan (1993). O passo espectral é um quociente de Rayleigh relacionado com uma média da matriz Hessiana. Esta escolha do tamanho do passo requer pouco esforço computacional e aumenta a velocidade de convergência dos métodos de gradientes projetados.

\section{MODELAGEM ESTOCÁSTICA IMPLÍCITA}

Os modelos estocásticos explícitos incorporam no próprio problema as incertezas. No caso do problema de despacho hidrotérmico, usam distribuição de probabilidades para vazões diretamente no problema, desta forma a solução do problema já fornece uma política ótima. Por outro lado, os modelos estocásticos implícitos, não consideram as incertezas diretamente no problema. A abordagem adotada nesse trabalho é determinar uma política ótima de despacho hidrotérmico utilizando modelagem estocástica implícita, ou seja, primeiramente são geradas séries sintéticas não equiprováveis, respeitando casos de séries com eventos de cheias e secas e para cada série sintética gerada resolve se um problema determinístico. Nesse trabalho, foram resolvidos 200 problemas do despacho hidrotérmico, ou seja, como dados de entrada foram utilizadas 200 séries sintéticas geradas a partir da metodologia descrita em (Detzel, et.al, 2014). Cada um dos 200 problemas de otimização foi resolvido utilizando os métodos Lagrangeano Aumentado combinado com o método de Gradiente Espectral Projetado para resolver os subproblemas gerados a cada iteração.

\section{SISTEMA TESTE E RESULTADOS}

A metodologia atual adota a hipótese simplificadora de agregação dos reservatórios que operam em uma mesma região, no entanto, estas simplificações podem omitir características importantes do sistema, tornando-o pouco flexível e colocando em risco a confiabilidade dos resultados. Para os testes apresentados nesse trabalho foram utilizados séries sintéticas hidrológicas geradas a partir da metodologia apresentada em (Detzel et al., 2014).

O sistema teste considerado é composto por 111 usinas hidráulicas e 32 usinas térmicas, distribuídas em cinco subsistemas. Desses subsistemas, quatro seguem as características dos subsistemas Sul, Sudeste, Nordeste e Norte e o quinto é formado somente pela usina Itaipu, com a consideração de possuir somente geração e não possuir demanda.

O problema formulado com essas características tem 22.560 variáveis e 13.620 restrições, sendo que as restrições de caixa não foram consideradas, pois elas não foram penalizadas e o número de variáveis representa apenas as variáveis relacionadas ao problema, não sendo considerados na contagem os multiplicadores de Lagrange.

Foram consideradas 200 séries sintéticas representando uma gama de situações hidrológicas (Detzel et al., 2014). Dessa maneira, são resolvidos 200 problemas do despacho hidrotérmico com a finalidade de validar a metodologia proposta.

Todas as rotinas foram programadas em MATLAB 2010a. Para agilizar o processamento das 200 séries sintéticas foi utilizado um cluster composto por um head-node (nó de controle) e 18 worker-nodes (nós de processamento). Cada um dos nós do cluster é composto por dois processadores com seis núcleos de processamento físico e mais seis núcleos de processamento lógico o que totaliza um poder de processamento de 24 núcleos por nó, totalizando 432 núcleos de processamento. A distribuição das séries nos núcleos de processamento foi feita por meio de um Job, o qual foi responsável por alocar seis núcleos de processamento para cada uma das séries, sendo que para cada conjunto o código foi o mesmo, variando apenas os dados de entrada.

Dos 200 problemas resolvidos, o Lagrangeano Aumentado e o SPG obtiveram solução de 128 problemas. Quando analisada a condição hidrológica do restante dos problemas constata-se que não eram favoráveis, ou seja, as afluências eram baixas e a geração das térmicas não era suficiente para suportar os períodos de seca. Para ilustrar esse fato, foram 
selecionados dois problemas, o primeiro foi resolvido utilizando os dados da série sintética 4, onde ocorreu a convergência (Fig. 1) e o segundo foi resolvido utilizando os dados da série sintética 186, que não convergiu (Fig. 2).

Nas figuras Fig. 1 e Fig. 2 estão representadas a soma por mês da geração térmica (GT), da geração hidráulica (GH) e da afluência, dessa pode-se analisar o comportamento das gerações em afluências altas e baixas.

A série sintética 186 possui períodos de pouca afluência, com isso quando o problema é resolvido a demanda não é atendida, pois as usinas térmicas consideradas no sistema não possuem potência suficiente para complementar a geração das usinas hidráulicas.

Por outro lado, analisando o despacho do problema utilizando a série sintética 4, o qual tem períodos de afluências altas, pode-se notar que a geração térmica atua como complemento da geração hidráulica nos períodos onde a afluência diminui.

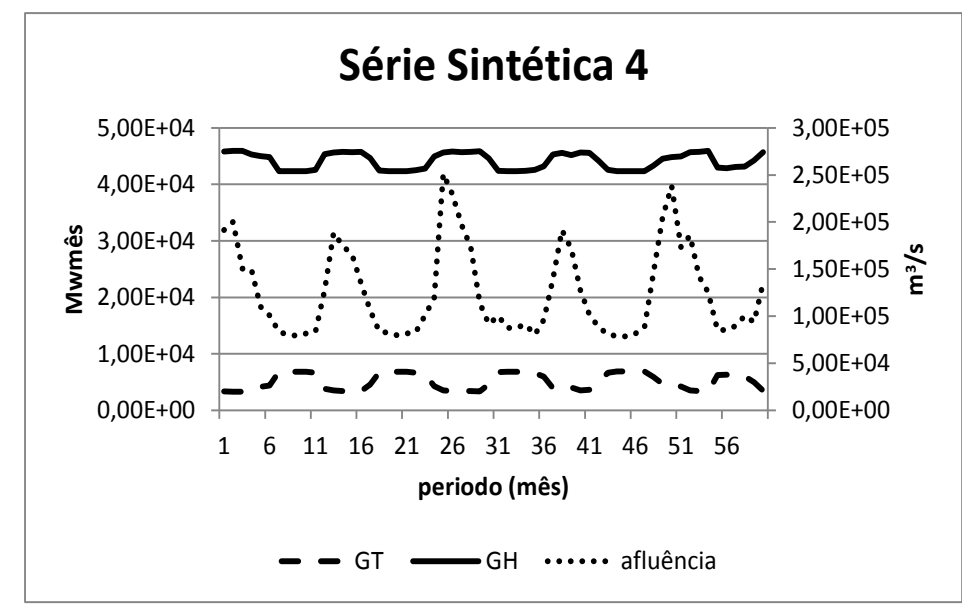

Figura 1: Despacho hidrotérmico da série sintética 4

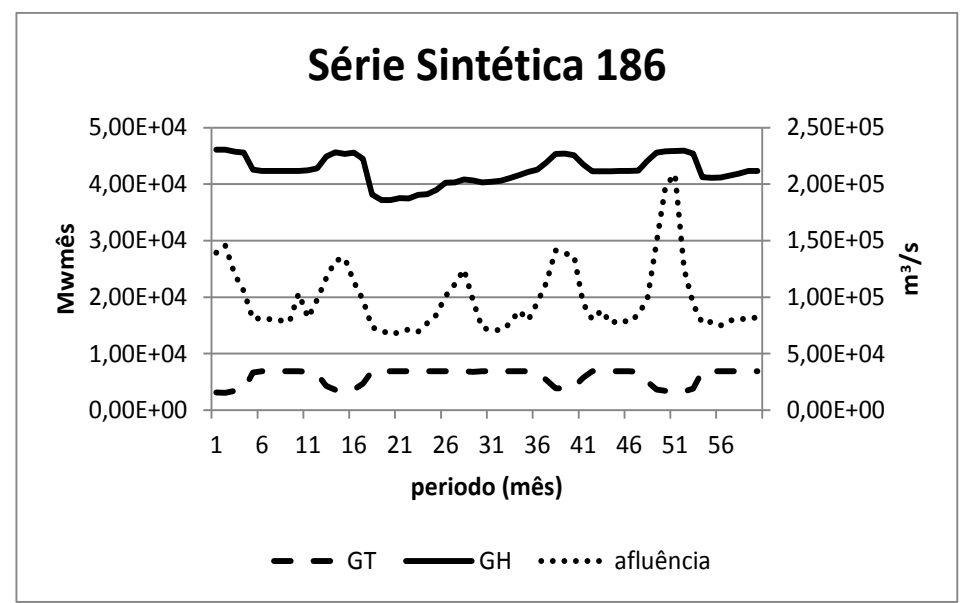

Figura 2: Despacho hidrotérmico da série sintética 186

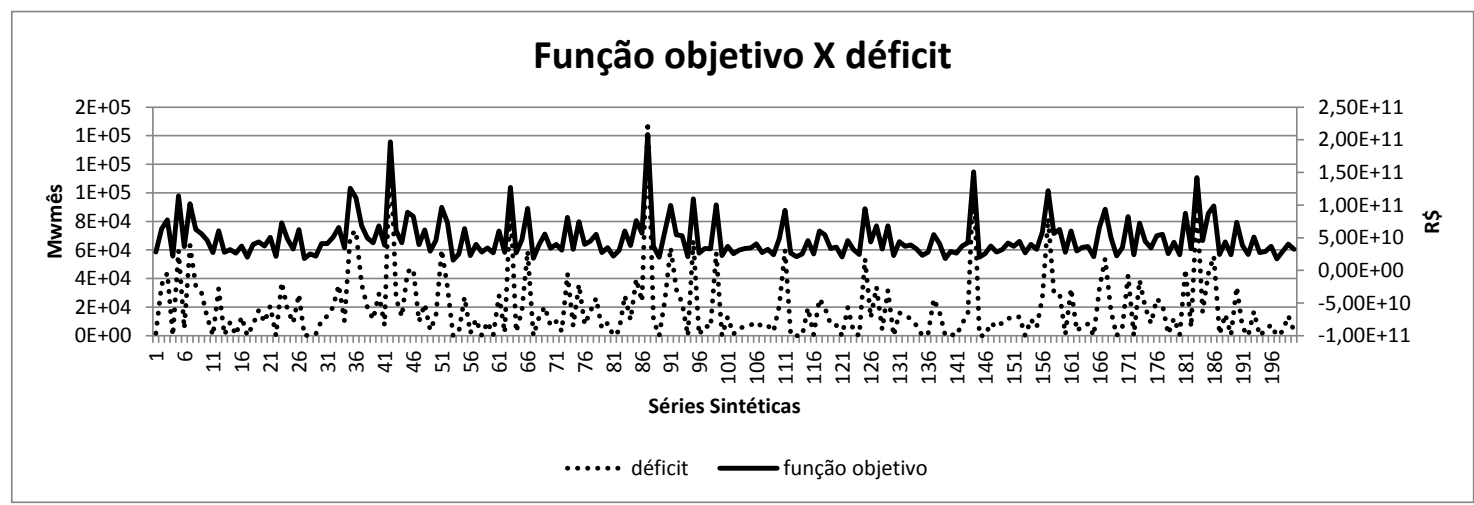

Figura 3: Função objetivo versus déficit 
A Fig. 3 contém a soma da função objetivo e do déficit por série sintética. Analisando esse gráfico tem-se que quanto maior o déficit, maior o valor da função objetivo, confirmando a adequação da modelagem.

A média do tempo de processamento para cada problema foi de 5 horas, porém com o uso do cluster na distribuição das tarefas, o tempo total de processamento foi de 18 horas, o que viabiliza os estudos realizados.

Comparando os resultados apresentados aqui com os discutidos em (Kleina et al, 2012 e Marcilio et al , 2012) temse que os métodos Lagrangeano Aumentado e o SPG mostraram-se mais robustos, pois otimizaram problemas com dimensões maiores e com uma variedade maior de séries hidrológicas.

\section{CONCLUSÃO}

O método de Lagrangeano Aumentado juntamente com o método de Gradiente Espectral Projetado mostraram ser uma boa opção na resolução do problema de despacho hidrotérmico, o qual é, conforme descrito no artigo, de grande porte, com restrições lineares, não lineares, de caixa e não convexo. Por isso, é um problema de difícil solução e necessita de métodos robustos para resolvê-lo. Os testes computacionais realizados com o problema considerado, praticamente englobando todo o sistema interligado nacional brasileiro, foram satisfatórios e indicaram que a disponibilidade de recursos hidrológicos está inversamente relacionada com o tempo computacional, ou seja, quanto maior o recurso disponível menor o tempo computacional.

Desta forma, a determinação de políticas ótimas de despacho hidrotérmico, utilizando a programação não linear com programação estocástica implícita, adotando uma representação detalhada das usinas hidráulicas e térmicas, mostrou se uma alternativa a ser considerada em futuros estudos.

\section{REFERÊNCIAS}

Benders J. F., "Partitioning Procedures for Solving Mixed-Variables Programming Problems", Numerische Mathematik, vol. 4, 1962, pp. 238-252.

Birgin E. G., Martínez J. M., “Augmented Lagrangian Method with Nonmonotone Penalty Parameters for Constrained Optimization", Computational Optimization and Applications, 2011.

Birgin E. G., Martínez J. M., and Raydan M., "Nonmonotone Spectral Gradient Methods on Convex Sets", SIAM Journal on Optimization, vol. 10, 2000, pp. 1196-1211.

Birgin E. G., Martínez J. M., and Raydan M, "SPG: Software for Convex Optimization", ACM Transactions on Mathematical Software, vol. 27, 2001, pp. 340-349.

Borwein J. M., Barzilai J., "Two Point Step Size Gradient Methods", IMA Journal of Numerical Analysis, vol. 08, 1988, pp. 141-148.

Detzel, D. H. M.; Bessa, M. R.; Mine, M. R. M; Bloot, M. Cenários sintéticos de vazões para grandes sistemas híbridos através de modelos contemporâneos e amostragem Revista Brasileira de Recursos Hídricos, 19:17-28,2014..

Grippo L., Lampariello F. and Lucidi S., "A Nonmonotone Line Search Technique for Newton's Method”, SIAM Journal on Numercial Analysis, vol. 23, 1986, pp. 707-716.

Kleina M., Marcilio D.C, Oening A.P., Bessa, Matioli L.C, O Método de Pontos Interiores Aplicado ao Problema do Despacho Hidrotérmico. CNMAC2012, pp 81-87-1168, 2012

Luenberger D. G., Linear and Nonlinear Programming, Springer, Nova York, 2005.

Marcilio D.C, Oening A.P., Bessa, M.R., Matioli L.C., Kleina M., M. Bloot, Pontos Interiores versus Lagrangeano Aumentado na Otimização do Despacho Hidrotérmico. CNMAC2012, pp 1162-1168, 2012.

Nocedal J., Wright S., Numerical Optimization. Springer, Nova York, 2005.

Pereira M. V. F., "Optimal Stochastic Operations Scheduling of Large Hydroelectric Systems", International Journal of Electrical Power \& Energy Systems, vol. 11, 1989, pp. 161-169.

Pereira M. V. F. and Pinto L. M. V. G., "Stochastic optimization of a Multireservoir Hydroelectric Systems: a Decomposition Scheduling”, Water Resources Research, vol. 21, 1985, pp. 779-792.

Raydan M. "On the Barzilai and Borwein choice of Steplenght for the Gradient Method", IMA Journal of Numerical Analysis, vol. 13, 1993, pp. 321-326.

Rockafellar R. T, A dual approach for solving nonlinear programming problems by unconstrained optimization, Mathematical Programming, vol. 5, 1973, pp. 354-373. 


\title{
AUGMENTED LAGRANGEAN AND SPECTRAL PROJECTED GRADIENT METHODS APPLIED TO SOLVING THE HYDROTHERMAL DISPATCH PROBLEM
}

\author{
Luiz Carlos Matioli, matioli@ufpr.br ${ }^{1}$ \\ Débora Cíntia Marcilio, deboramarcilio@gmail.com²
}
${ }^{1}$ Federal University of Paraná - Departament of mathematics/PPGM - 81531-990, Centro Politécnico, Curitiba, PR
${ }^{2}$ Federal University of Paraná - PPGMNE - 81531-990, Centro Politécnico, Curitiba, PR

\begin{abstract}
In this work a methodology for determination of hydrothermal dispatch, which uses nonlinear programming with implicit stochastic modeling, adopting detailed representation of hydro and thermal power plants was developed. The modeling of the hydrothermal dispatch problem results in a problem of nonlinear programming of large, nonconvex, differentiable and non-linear equality constraints, linear equality and inequality and box constraints. Thus, a methodology has been developed based on Augmented Lagrangian and Spectral Projected Gradient methods, which are known to have good convergence properties, robustness, compactness, easy to implement and require only firstorder information. To validate the methodology, was used a well representative system of the National Interconnected System-SIN, with 111 hydro plants and 32 thermal power plants, distributed over five subsystems. The results showed to be satisfactory, both from the perspective of the problem as the order of computational performance. That said, the methodology developed showed promising in the determination of economic dispacth, sinse results were consistent with reality and the methods of Augmented Lagrangian and Gradient Spectral Projected Gradient shown to be robust and stable.
\end{abstract}

Keywords: Augmented Lagrangian, Spectral Projected Gradient, hydrothermal dispatch and Optimization. 